\title{
Protective Effect of KTMon Streptozotocin-Induced Diabetic Symptoms and Their Potential Mechanisms in Rats
}

\author{
Baozhong Diao ${ }^{1}$, Weirong Jin ${ }^{1, ~ *}$, Feng E. Zhang ${ }^{2}$, Wenzhou Zhang ${ }^{3}$ \\ ${ }^{1}$ Department of Formulation Branch, Liaocheng People's Hospital, Liaocheng, China \\ ${ }^{2}$ Department of Emergency, Liaocheng People's Hospital, Liaocheng, China \\ ${ }^{3}$ Department of Pharmacy, Liaocheng People's Hospital, Liaocheng, China
}

Email address:

lcsrmyyjwr@163.com (Weirong Jin)

${ }^{*}$ Corresponding author

To cite this article:

Baozhong Diao, Weirong Jin, Feng E. Zhang, Wenzhou Zhang. Protective Effect of KTMon Streptozotocin-Induced Diabetic Symptoms and Their Potential Mechanisms in Rats. American Journal of Clinical and Experimental Medicine. Vol. 6, No. 3, 2018, pp. 64-68. doi: 10.11648/j.ajcem.20180603.11

Received: March 26, 2018; Accepted: April 12, 2018; Published: May 24, 2018

\begin{abstract}
The present study aimed to evaluate the therapeutic effects of ketangmin (KTM) on streptozotocin-(STZ) induced diabetic symptoms and their potential mechanisms. Methods: The effect of KTM on body weight, blood glucose, damaged pancreafic $\beta$-cells, oxidative stresses, proinflammatory cytokines, and glucose metabolizing enzymes in liver was studied. Results: The results show that administration of KTM can restore abnormal oxidative indices near normal levels. The STZ-damaged pancreatic $\beta$-cells of the rats were partly recovered gradually after the mice were administered with KTM 6 weeks later. Therefore, we may assume that KTM is effective in the protection of STZ-induced diabetic rats and KTM may be of use as antihyperglycemic agent.
\end{abstract}

Keywords: Ketangmin (KTM), Streptozotocin-Induced Diabetic Symptoms, Potential Mechanisms

\section{Introduction}

Diabetes mellitus is linked to a series of cardiovascular and micro vascular changes that can be manifest as a wide range of complications [1]. Diabetes mellitus is a metabolic disorder affecting carbohydrate date, fat, and protein metabolism. Long-standing di-abetes is prone to various complications which include cardiac, kidney, eye problems and pancreatic cancer [2, 3]. More effective and safer treatment modalities for diabetes mellitus have to be investigated. KTM is developed by our hospital, new kind antidiabetic preparation, More than 10 years of clinical applications. For diabetes has a good effect of prevention and control of diabetes and complication. Up to now, however, no detailed investigation has been carried out on the effective constituents of KTM for an-tihyperglycemic activities. Today, calling for return to natural therapy to treat diabetes stronger and stronger [4].

In the present study, the purpose is to focus on theKTM for seeking new Traditional Chinese medicine preparation to alleviate the diabetesmellitus.

\section{Materials and Methods}

\subsection{KTM Composition}

KTM composition is as follows: Astragalus membranaceus (Fisch.) Bge. var. mongholicus (Bge). Hsiao (150g); Atractylodes macrocephala Koidz (150g); Trichosanthes kirilowii Maxim. (300g); Rehmannia glutinosa Libosch (300g); Dioscorea opposita Thunb (300g) eleven herbs Etc.

Above 8 herbs were heated and boiled in water twice: first, for two hours and, second, for 1.5 hours. Then follow these steps: the merger decoction, filtration, the filtrate being concentrated to about $800 \mathrm{~mL}$, adding water to $1000 \mathrm{~mL}$, mixing, packaging, and sterilization.

\subsection{Animals}

Healthy male adult Wistar rats (2 months old and weighing $200 \pm 20 \mathrm{~g}$ ) were used in the study. This study was carried out in accordance with the Guide for the Care and Use of 
Laboratory Animals. Care was taken to minimize discomfort, distress, and pain to animals. The protocols were approved by the Hospital Ethics Committee. Experimental diabetes was induced by intrapulmonary (i.p.) injection with a freshly prepared solution of STZ (Sigma, USA) dissolved in citrate buffer ( $\mathrm{pH} 4.5$ ) at the dose of $35 \mathrm{mg} / \mathrm{kg}$ body weight. Only rats with blood glucose concentration more than $240 \mathrm{mg} / \mathrm{dL}$ were considered diabetic and utilized for the study Glucose level was assessed by using enzymatic glucose oxidase peroxidase commercially available kit method, $72 \mathrm{~h}$ after STZ induction. The rats with blood glucose concentration more than 240 $\mathrm{mg} / \mathrm{dL}$ were considered diabetic and used for the study.

\subsection{Treatment Schedule and Experimental}

Protocol. Forty hypoglycemic rats were se-lected and allocated equally into 4 groups and administered orally Saline, KTM (10 g/kg/d), KTM (20 mg/kg/d), and KTM (30 mg/kg/d) respectively. The other 10 normal rats were administered orally with the saline and used as the control group.

Body weight of all animals was recorded on 0 , 1st, 2nd, 3rd, 4 th, 5th, and 6th week of treatment. Blood of all animals was collected through retro orbital route initially and on 6thweek of treatment to measure the serum glucose levels. Then, the rats were killed. The blood sample had a right to clot for 20 minutes at refrigerator temperature. The blood samples were then shifted to clean centrifuge tubes. Lithium heparin was in addition to obtain plasma. The withdrawn blood was isolated by centrifugation at $4000 \mathrm{rpm}$ for 10 minutes to obtain serum. The serum was stored in a freezer until analysis. The liver was dissected out for the measurement of IL-1ßand TNF-a. The pancreas was reserved for pathological histology using hematoxylin and eosin (H\&E) staining.

\subsection{Measurement of IL-1ß and TNF-a Level in Liver}

The liver was dissected out for the measurement of hepatic glycogen. The liver TNF-a and IL-1 $\beta$ were measured using a commercial enzyme-linked immunosorbent assay (ELISA) kit (Shanghai Jinma Biological Technology Inc., China) following the manufacturer's instruction.

\subsection{Measurement of Lipid Profile}

Total cholesterol (TC), triglycerides (TAG), low-density lipoprotein (LDL) cholesterol, and high-density lipoprotein (HDL) cholesterol was determined in the serum samples using commercially available kits (Shanghai Jinma Biological Technology Inc., China).

\subsection{Measurement of Glucose Metabolizing Enzymes}

The liver homogenate was used to assess metabolism enzymes. Glutamic oxaloacetate transaminase (GOT), glutamate pelvic transaminase (GPT), and lactate dehydrogenase (LDH) were measured using commercially available kits (Shanghai Jinma Biological Technology, Inc., China).

\subsection{Estimation of the Total Antioxidant Activity}

Total antioxidant status (TAOS) of hepatic tissue was determined by the way introduced by Laight et al. [9]. The increase in absorbance at $405 \mathrm{~nm}$ was measured by using a microplate reader (Shanghai Xunda Medical Technology, Inc., China).

\subsection{Statistical Analysis}

All data were analyzed by a one-way analysis of variance, and the differences between means were established by Newman-Keuls test. The datum represents means and standard deviations. The significant level of $5 \%(\mathrm{P}<0.05)$ was considered to be the minimum acceptable probability for the difference between the means.

\section{Results and Discussion}

The objective of this study was to investigate whether the polysaccharides from 1. Obli-quus (KTM) could produce hypoglycemic activity in STZ-induced diabetic rats. STZ is an antibiotic extracted from Streptomyces chromosomes and is diabetogenic due to a selective cytotoxic action upon pancreatic $\beta$-cell [5]. In the present investigation, STZ injected rats exhibit clinic pathological features including biochemical, oxidative, and metabolic changes. These changes were halted in KTM treated animals. Many studies have shown a glycemia and decreased body association between hyper weight of diabetic animals [6]. As showed in Figure 1, the STZ-treated animals had significantly reduced body weight than the control rats $(\mathrm{P}<0.01)$. When compared with TZ-treated animals, the body weight gains were significantly increased in groups of KTM treated animals $(\mathrm{P}<0.05 ; \mathrm{P}<0.01)$ in a dose-dependent manner. STZ in the experimental diabetic model leads to defective glu-cose oxidation and causes hyperglycemia [7]. Our study is consistent with this report. The blood glucose level in normal rats remained constant for six weeks and was signifi-cantly $(\mathrm{P}<0.01)$ lower than those of streptozotocininduced diabetic rats (Table 1). Upon treatment with KTM for six weeks, the blood glucose levels of all diabetic rats were mar-kedly diminished in a dose-dependent manner, suggesting that KTM is a potent thera-peutic agent against diabetes.

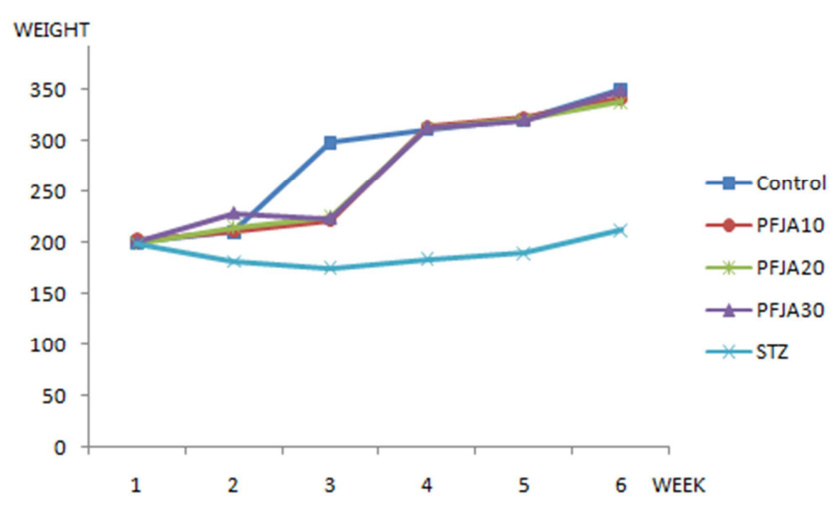

Figure 1. Effect of PFJA administration on diabetic rats body weight values represent the mean \pm SEM. $\# p<0.05$ versus $S T Z$ group.

Table 1. Effect of KTM on blood glucose levels in STZ-hyperglycemic rats. $(n=10)$ 


\begin{tabular}{ll}
\hline Different groups & Blood glucose $(\mathbf{m m o L} / \mathbf{L})$ \\
\hline Control & $5.9 \pm 1.2$ \\
STZ & $22.2 \pm 2.2$ \\
KTM30 & $10.1 \pm 3.2^{*}$ \\
KTM20 & $15.5 \pm 2.0^{*}$ \\
KTM10 & $18.6 \pm 3.0^{*}$ \\
\hline
\end{tabular}

Mark: Values are means $\pm \mathrm{SEM} ; \mathrm{n}=10 . * \mathrm{P}<0.05$ versus $\mathrm{STZ}$ group

Hypoglycemic mechanisms of many polysaccharides are closely related to their an-tioxidant activity [8]. Hence, it is plausible that the hypoglycemic effect of KTM may be explained by the effect on alleviating oxidative stress. The TAOS is a sign of O2- and other oxidant species. We measured TAOS activity as an indirect indication of the formation of $\mathrm{O} 2$-and other oxidant species. Results of hepatic TAOS are shown in Table 2. The STZ treatment increased TAOS. TAOS in the KTM-20-and KTM-30-treated groups were sig-nificantly lower than those in the STZ-treated group $(\mathrm{P}<0.01$, rep. $)$.

Table 2. Effect of KTM on TAOS activity ( $\mu M L$-ascorbate). $(n=10)$.

\begin{tabular}{ll}
\hline Groups & TAOS activity( $\boldsymbol{\mu M L}$-ascorbate) \\
\hline STZ & $82.00 \pm 3.31$ \\
KTM(10mg) & $68.36 \pm 2.39^{*}$ \\
KTM(20mg) & $60.02 \pm 1.32^{*}$ \\
KTM (30mg) & $51.01 \pm 2.20^{*}$ \\
Control group & $28.04 \pm 1.02$ \\
\hline
\end{tabular}

Mark: Values are means $\pm \mathrm{SEM} ; \mathrm{n}=10 . * \mathrm{P}<0.01$ versus $\mathrm{STZ}$ group

It has been observed that over $75 \%$ of premature deaths in diabetes are related to coronary artery disease caused by abnormal lipid metabolism, which often leads to altered lipid profile of the victim [9]. Lipid peroxidation is part of the characteristic features of chronic diabetes. The increased free radicals produced may react with polyunsaturated fatty acids in cell membranes leading to lipid peroxidation. It will, in turn, result in the elevated pro-duction of free radicals [10]. In the present experiment, significantly increased lipid perspiration products were observed in STZ-induced diabetic rats. Treated with KTM-20 and KTM-30 for 6 weeks, LDL level was reduced $(\mathrm{P}<0.05)$, whereas HDL cholesterol was raised $(\mathrm{P}<0.05)$ (Table 3). These results further confirm that there is a strong correlation between oxidative stress and diabetes occurrence.

Table 3. Effect of KTM on changes in the levels of serum lipid profile. $(n=10)$.

\begin{tabular}{llllll}
\hline Lipid profile $\mathbf{~ m m o l} / \mathbf{L}$ & Cholesterol & Triglycerides & LPO & HDL & LDL \\
\hline Control & $4.30 \pm 0.22$ & $0.81 \pm 0.17$ & $8.59 \pm 0.11$ & $0.72 \pm 0.63$ & $0.28 \pm 0.01$ \\
STZ & $10.55 \pm 1.01$ & $2.22 \pm 0.91$ & $15.20 \pm 1.30$ & $1.34 \pm 0.21$ & $0.70 \pm 0.04$ \\
KTM10 & $8.31 \pm 1.60^{*}$ & $0.99 \pm 0.05^{*}$ & $9.29 \pm 0.33^{*}$ & $0.82 \pm 0.07^{*}$ & $0.37 \pm 0.31^{*}$ \\
KTM20 & $7.31 \pm 0.38^{*}$ & $0.81 \pm 0.03^{*}$ & $8.86 \pm 0.01^{*}$ & $0.86 \pm 0.05^{*}$ & $0.32 \pm 0.22^{*}$ \\
KTM30 & $4.30 \pm 0.21^{*}$ & $0.78 \pm 0.07^{*}$ & $7.39 \pm 1.22^{*}$ & $0.72 \pm 0.03^{*}$ & $0.23 \pm 0.04^{*}$ \\
\hline
\end{tabular}

Mark: Values are shown as means \pm SEM; ${ }^{*} \mathrm{p}<0.01$ versus $\mathrm{STZ}$ group.

It was suggested that the STZ-induced weight loss in animals was the result of protein wasting in a situation of unavailability of carbohydrate for utilization as an energy source [11]. In diabetes, cytoplasmic enzymes such as GOT, GPT, and LDH pass into blood plasma and their activities in serum increase [12]. In the present study of oral treatment of KTM-20 and KTM-30 significantly $(\mathrm{P}<0.05)$ restored the altered glycoprotein components of diabetic rats in a dose dependent manner (Table 4).

Table 4. Effect of KTM on GOT, GPT, and LDH. (Unit $L^{-1}, n=10$ ).

\begin{tabular}{llll}
\hline Group & GPT & GOT & LDH \\
\hline Control & $66.0 \pm 2.27$ & $79.8 \pm 0.87$ & $48.6 \pm 0.11$ \\
STZ & $159.2 \pm 1.01$ & $288.2 \pm 0.91$ & $317.2 \pm 3.30$ \\
KTM10 & $155.1 \pm 1.60^{*}$ & $184.9 \pm 0.05^{*}$ & $299.8 \pm 0.33^{*}$ \\
KTM20 & $129.1 \pm 0.38^{*}$ & $103.7 \pm 0.03^{*}$ & $186.4 \pm 0.01^{*}$ \\
KTM30 & $67.1 \pm 0.21^{* *}$ & $80.8 \pm 0.07^{* *}$ & $77.3 \pm 1.22^{* *}$ \\
\hline
\end{tabular}

Mark: Values are shown as means $\pm \mathrm{SEM} ; * \mathrm{P}<0.01 \mathrm{P}$ versus $\mathrm{STZ}$ group.

A chronic inflammation may play a role in the pathogenesis of metabolic disorders [13, 14]. Prospective studies have identified proinflammatory cytokines as predictors of di-abetes [15]. TNF-a was the first proinflammatory cytokine implicated in pathogenesis of obesity-related insulin resistance and diabetes [16] and studies conducted with IL-1ßantagonism beneficial effects on glycine hemoglobin and $\beta$-cell function [17]. Therefore, the effect of KTM on TNF- $\alpha$ and IL-1 $\beta$ production was determined by ELISA. In comparison to STZ group (Figure 2), treatment with KTM-30 led to a marked de-crease in IL-11ßlevels $(\mathrm{P}<0.05)$. In addition, KTM-30 suppressed STZ-induced TNF- $\alpha$ production $(\mathrm{P}<0.05)$ (Figure 3$)$. 


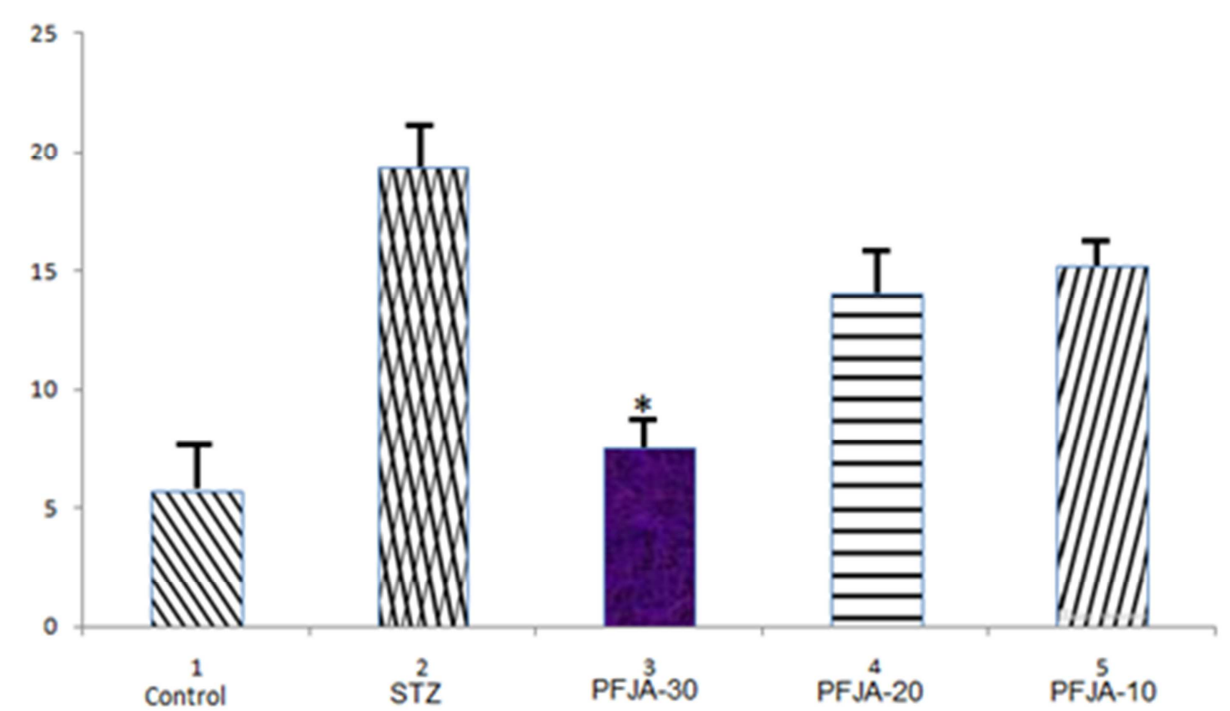

Figure 2. Effect of PFJA on IL-1Blevel. Values represent the mean $\pm S E M .{ }^{*} p<0.05$ versus STZ group.

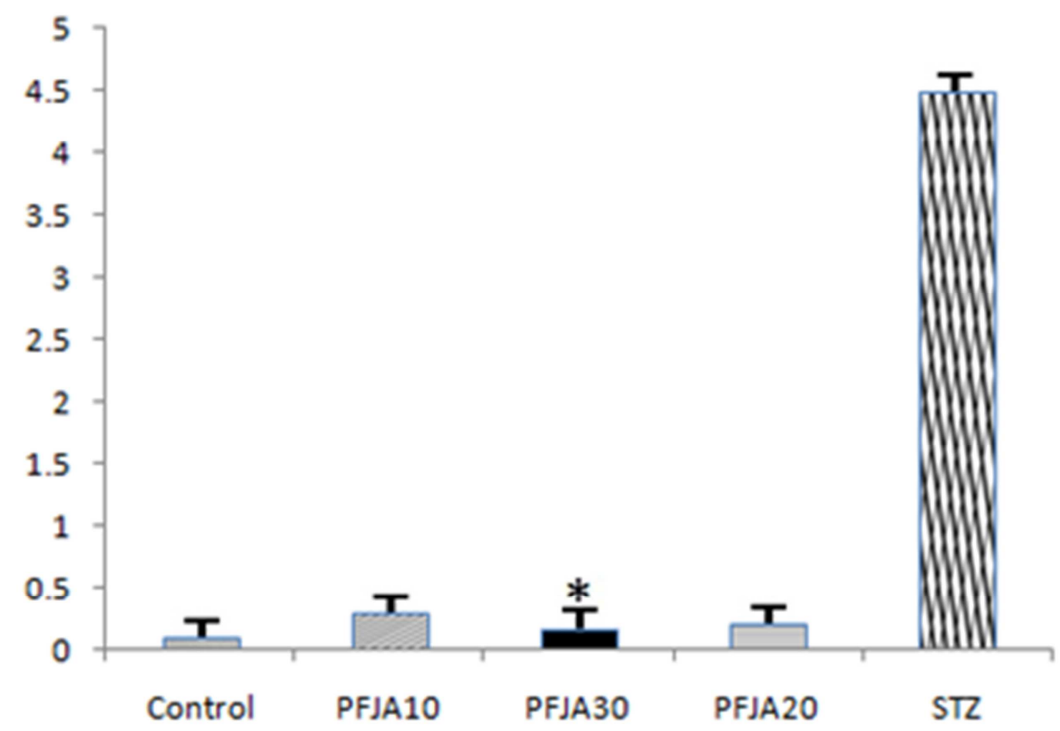

Figure 3. Effect of PFJA on TNF- $\alpha$ level. Values represent the mean $\pm S E M .{ }^{*} p<0.05$ versus $S T Z$ group.

STZ is a compound commonly used to cause diabetes in rodents. The mode of its action is mediated through the induction of severe damages to the $\beta$-cells [18]. The protective effect of KTM against the damages to $\beta$-cells induced by STZ toxicity was studied. Selective destruction of pancreatic $\beta$-cells by STZ in the experimental diabetic model was observed (Figure 4(b). We observed focal necrosis, congestion in central vein, and infiltration of lymphocytes in the pancreas

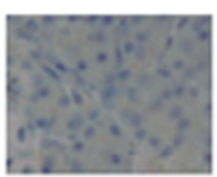

(a. Control $\times 100)$

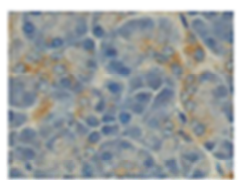

(b. STZ $\times 1 \infty$ )

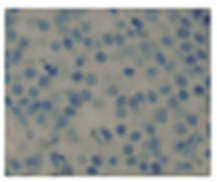

(C. PFJA30 *100)

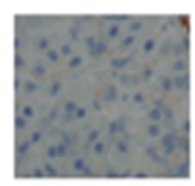

(d. PFJA20 * 10 )

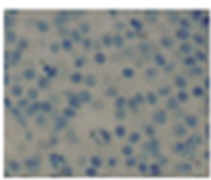

(e. PFJA10 $* \infty)$

Figure 4. Iset cell death and replication represented by hematoxylineosin. The islet cells of diabetic rat of STZ treatment(b)showed extensive cell lysis, representing loss ofplasma membrane wih condensed nulei and dissolved cytoplasm in wideintercellular spaces. In constrast, the islet cell of PFJA-fed rat(c, $d$, e) was partly recovered. (c) Islet from a normal rat. 
In summary, we have demonstrated that KTM has therapeutic effects against diabetes via multiple pathways. It displays antioxidant actions, hyperlipidemia activity, and protects the pancreas from diabetes induced injuries in STZ-treated rats. Therefore, KTM may provide a valuable therapeutic option against diabetes.

\section{Acknowledgements}

This work was supported by Liaocheng People's Hospital Pharmacy Department and Liaocheng project "Research on KTM development and quality standards". There was no funding.

\section{Conflict of Interest}

The authors declare no financial or other conflicts of interest.

\section{References}

[1] Frank L. Bowling, S. Preventing and treating foot complications associated with diabetes mellitus. Nature Reviews Endocrinology 11,606-616, (2015)

[2] T. Vetrichelvan, M. Jegadeesan, and B. A. U. Devi. "Anti-diabetic activity of alcoholic extract of Celosia argentea LINN. seed in rats," Biological and Pharmaceutical Bulletin, vol. 25 , no. 4 , pp.526-528, 2002.

[3] Naoto Egawa, Yingsong Lin, Taku Tabata et al., ABO blood type, long-standing diabetes, and the risk of pancreatic cancer. World J Gastroenterol. 2013 Apr 28; 19(16): $2537-$ 2542 .

[4] Bharti SK, Krishnan S, Kumar A. Phytotherapy for diabetes mellitus: back to nature. Minerva Endocrinol. 2015 Nov 12.

[5] Szarka A1, Horemans N, Passarella S et al. Demonstration of an intramitochondrial invertase activity and the corresponding sugar transporters of the inner mitochondrial membrane in Jerusalem artichoke (Helianthus tuberous L.) tubers. Planta. 2008 Oct; 228(5):765-75.

[6] M. W Massing, C. A. Sueta, M. Chowdhury, D. P. Biggs, and R. J. Simpson Jr., "Lipid manage-ment among coronary artery disease patients with diabetes mellitus or advanced age," The American Journal of Cardiology, vol. 87, no. 5, pp. 646-649, 2001.

[7] S. A. Metz, "Oxygenation products of antimonic acid: third messengers of insulin release," Prostaglandins, vol. 27, pp. 147-151, 1984.

[8] V Chen and C. D. Ianuzzo, "Dosage effects of streptococci on rat tissue enzyme activities and glycogen concentration; Canadian Journal of Physiology and Pharmacology, vol. 60, no. 10, pp. 1251-1256,1982.

[9] A. R. Chaudry, M. Alam, M. Ahmad, F. Z. Khan, and N. Nomani, "Studies on medicinal herbs. II: effect of Colchicum luteumonbio chemical parameters of rabbit serum; Fitoterapia, vol. 64 , no. 6 , pp. 510-515, 1993.

[10] M. Y Donath and S. E. Shoelson, "Type 2 diabetes as an inflammatory disease; Nature Re-views Immunology, vol. 11, no. 2, pp. 98-107, 2011.

[11] A. Chawla, K. D. Nguyen, and Y P. S. Goh, "Macrophage mediated inflammation in metabolic disease; Nature Reviews Immunology, vol. 11, no. 11, pp. 738-749, 2011.

[12] J. Spranger, A. Kroke, M. Mohlig et al., "Inflammatory cytokines and the risk to develop type 2 diabetes: results of the prospective population-based European Prospective Investigation into Cancer and Nutrition (EPIC)-Potsdam study" Diabetes, vol. 52, no. 3, pp. 812-817, 2003.

[13] G. S. Hotamisligil, P. Arner, J. F. Caro, R. L. Atkinson, and B. M. Spiegelman, "Increased adi-pose tissue expression of tumor necrosis factor-a in human obesity and insulin resistance," Journal of Clinical Investigation, vol. 95, no. 5, pp. 2409-2415, 1995.

[14] C. Cavelti-Weder, A. Babians-Brunner, C. Keller et al., "Effects of grievously on glycemia and inflammatory markers in type 2 diabetes; Diabetes Care, vol. 35, no. 8, pp.1654-1662, 2012. 